\title{
Management of C Shaped Canals in Mandibular Molars with a contemporary approach using MTA: Two Case Reports
}

\author{
Dr.Shristhi Sharma, Dr.AnantRaghav Sharma, Dr.Shanti Prasad Maurya, \\ Dr.SonaliChhabria
}

(MDS Endodontics, MBA, Senior Lecturer, Dept. Of Conservative Dentistry And Endodontics, Pacific Dental College And Research Centre,Bhillokabedla,Udaipur, India)

(MDS Periodontology, Reader, Dept. Of Periodontology,Pacific Dental College, Debari,Udaipur, India)

(MDS endodontics, senior resident dept. of dentistry, J L N medical college and hospital,Ajmer,India)

(Senior Lecturer, Dept. Of Prosthodontics, Pacific Dental College And Research Centre,Bhillokabedla,Udaipur, India)

Abstract:Knowledge of root canal anatomy with possible variations is of prime concern responsible forsuccessful endodontic therapy. Cooke and Cox were the first to document the C-Shaped canal in endodontic literature. The variation exhibits a fin or web connecting the individual root canals to form an orifice similar to letter ' $C$ '. The irregular areas in $C$-shaped root canal system often may harborsoft tissue remnant or infected bacterial biofilm, leading to inadequate cleaning and root canal obturation. The use of the ultrasonics, (MTA Fillapex), thermoplasticizedobturation techniques, SAF system, CBCT and Endodontic Microscopes can be successfully used to treat such cases. MTA based sealers help in proper sealing of the canal and also helps in regenerating bone and inducing cementogenesis.Recognition of unusual variations in the canal configurations is critical because it has been established that the root with a single tapering canal and apical foramen is the exception rather than the rule.

Keywords: $C$ shaped canal, Melton classification, MTA, MTAFillapex, fins and webs.

\section{INTRODUCTION}

Knowledge of root canal anatomy and variations between ethnic groups is essential for clinicians to facilitate effective root canal treatment (RCT) (Vertucci 1984). Of particular interest is the canal configuration of the mandibular second molar. Predicting and negotiating a $\mathrm{C}$-shaped root canal configuration is challenging.

The main feature of this variation is the presence of a fin or web connecting the individual root canals to form a letter ' $C$ ' shape at the root canal orifice. Since the first reports of c-shaped root canal configurations in 1979 by Cooke \& Cox, numerous investigations in this field have been conducted.

The failure of the Hertwig's epithelial root sheath to fuse on the lingual or buccal root surface was the main cause of a C-shaped root, which always contains a C-shaped canal.

It appears as a fusion between the distal and mesial roots, present either buccally or lingually. An intra-radicular ribbon shaped connection is present making this fusion irregular. This canal configurations provide a challenge for effective mechanical shaping, chemical cleansing and obtaining an impervious fluid tight seal of the entire root canal system. It is not only the configuration proving a challenge but also the unclear knowledge whether the $\mathrm{C}$ Shaped orifice at the pulp chamber actually continues till the apex. 
Table 1: The classification of C-shaped canals proposed by Fan et al.

Canal I (C1)-Continuous C-shaped root
canal from the orifice to the apex of the root

Canal II (C2) - One main root canal and a smaller one (;)

Canal III (C3) - Two or three root canals

Canal IV (CA) - An oval or a round canal

\section{Canal $V(C 5)$ - No canal lumen or there is one close to the apex}

Melton in 1991 proposed the following classification of C-shaped canals based on their cross sectional shape:Category I: Continuous C-shaped canal running from the pulp chamber to the apex defines a C-shaped outline without any separation.

Category II: the semicolon-shaped-orifice in which dentine separates a main C-shaped canal from one mesial distinct canal.

Category III: it refers to those with two more discrete and separate canals.

Subdivision I- C-shaped orifice in the coronal third that divides into two or more discrete and separate canals that join apically

Subdivision II- C-shaped orifice in the coronal third that divides into two or more discrete and separate canals in the midroot to the apex.

Subdivision III- C-shaped orifice that divides into two or more discrete and separate canals in the coronal third to the apex.

In this classification there has been no clear description of the difference between categories II and III as well as the clinical significance.

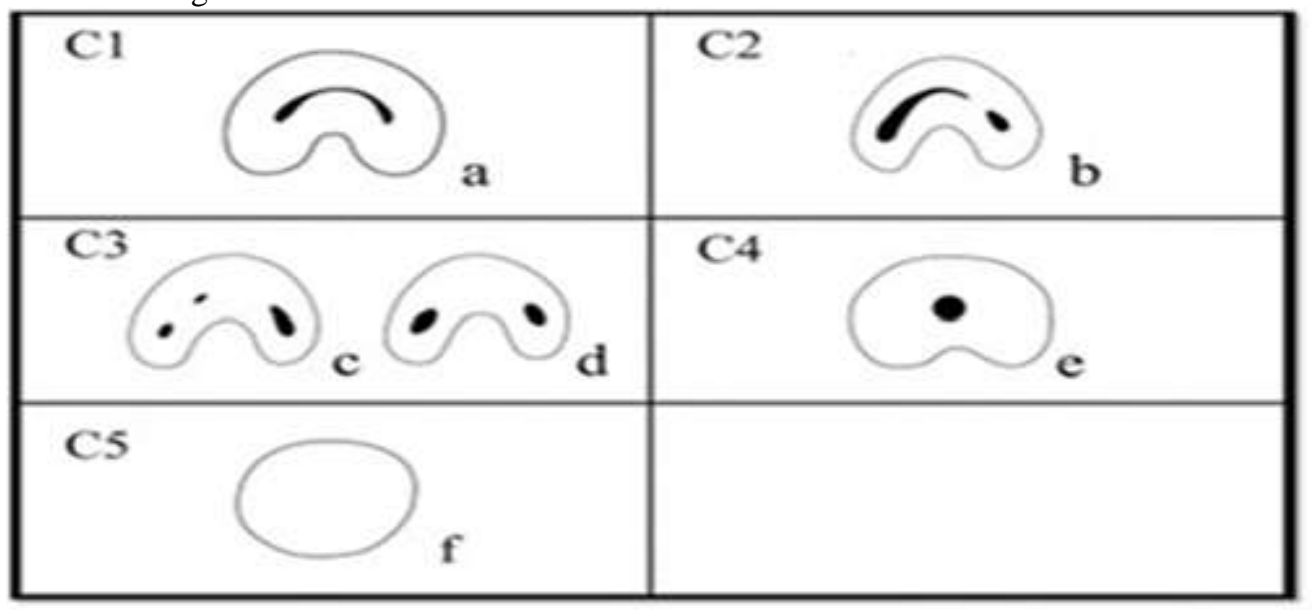

Type 1- Conical or square root with a vague, radiolucent longitudinal line separating the root into distal and mesial parts. There was a mesial and distal canal that merged into one before exiting at the apical foramen (a)

Type 2- Conical or square root with a vague, radiolucent longitudinal line separating the root into distal and mesial parts. There was a mesial and distal canal and the two canals appeared to continue on their own pathway to the apex (b)

Type 3-Conical or square root with a vague, radiolucent longitudinal line separating the root into distal and mesial parts. There was a mesial and distal canal one canal curved to and superimposed on this radiolucent line when running towards the apex and other canal appeared to continue on its own pathway to the apex (c)

Presence of these developmental abnormalities can alter the endodontic treatment and its prognosis. Therefore, it is necessary to diagnose their presence and evaluate the treatment intensity before the start. This manuscript 
Management of $C$ Shaped Canals in Mandibular Molars with a contemporary approach using MTA:...

reports the endodontic treatment of a maxillary left lateral incisor with periapical lesion that had a C-shaped anatomy.

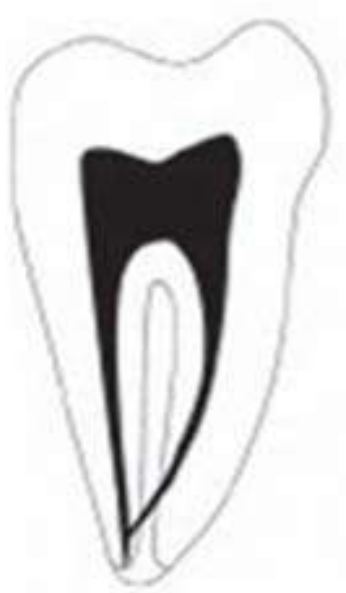

a

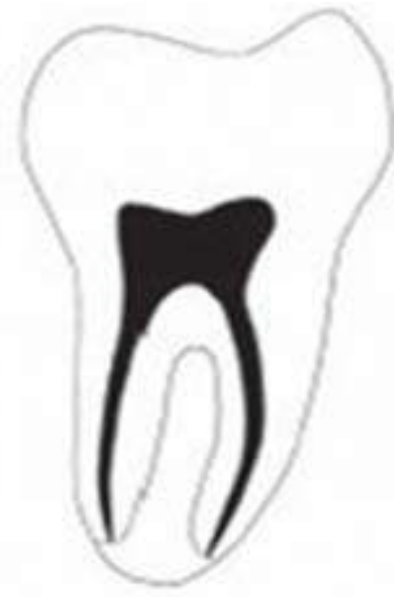

b

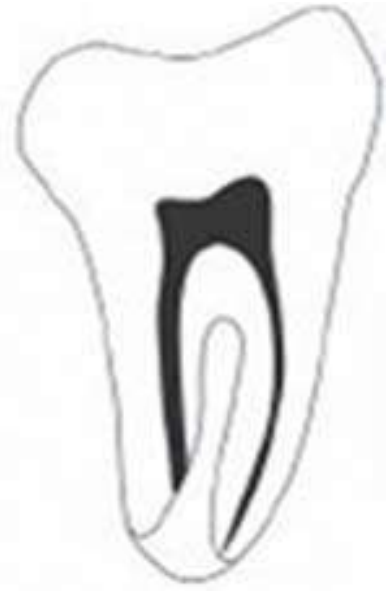

c

This article depicts 2 cases which were treated using MTA as an apical plug and MTA is used as a sealer to induce periapical bone formation and healing.

\section{Case Report I}

42 year old female patient reported to the department of endodontics with chief complaint of pain in relation to lower right molar for past 1 week. Patient gives history of night pain, sensitivity to thermal stimuli. Medical history of the patient was noncontributory. Clinical examination revealed the presence of large mesioocclusal carious lesion in relation to lower second molar. Vitality testing was conducted with electric pulp tester and showed exaggerated response. Preoperative radiograph revealed an occlusal radiolucency approaching pulp space. From the clinical and radiographic examination and result of vitality testing, a diagnosis of irreversible pulpitis was made and root canal treatment was planned. After attaining anaesthesia and rubber dam placement, an access cavity was prepared. After extirpation of pulp tissue from the chamber, the canal system appeared C shaped with 2 orifices. Working length was determined using apex locator (Propex,Dentsply)and confirmed using radiograph. The working length periapical radiograph revealed canal as Melton Category III. Passive ultrasonic irrigation was performed after apical preparation till 50 no. K file using cavitron ultrasonic unit. Ultrasonic irrigation helps to debride and disinfect the canals by acoustic streaming and cavitation, as the conventional techniques of cleaning and shaping cannot debride the webbed area of the $\mathrm{C}$ shaped canal.After biomechanical preparation of root canal, calcium hydroxide powder mixed with saline was applied and the access cavity was temporarily sealed with Coltosol (Coltene).

In the next appointment after 7 days, the patient had no pain and swelling. After rubber dam isolation, calcium hydroxide was removed with master apical file and CHX irrigation. The canal was filled by MTA fillapex as a sealer along with $2 \%$ guttapercha cones using a lateral condensation technique. One week after completion of endodontic treatment, patient had no clinical symptom and the tooth was restored with composite resin (Brilliant, Dentsply). At 12 month-follow up visit, the patient reported no symptoms and the radiograph showed healing of periapical lesion.

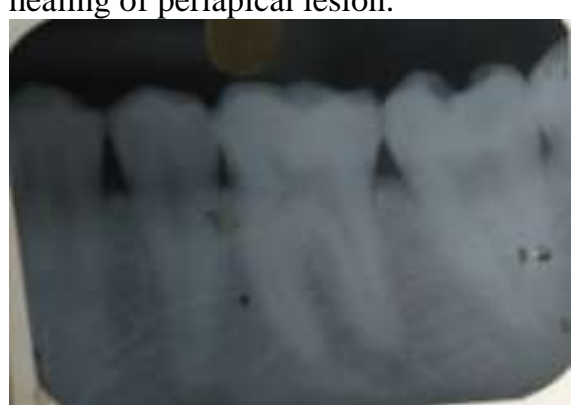

Fig 1: C shaped anatomy of pulpal chamber

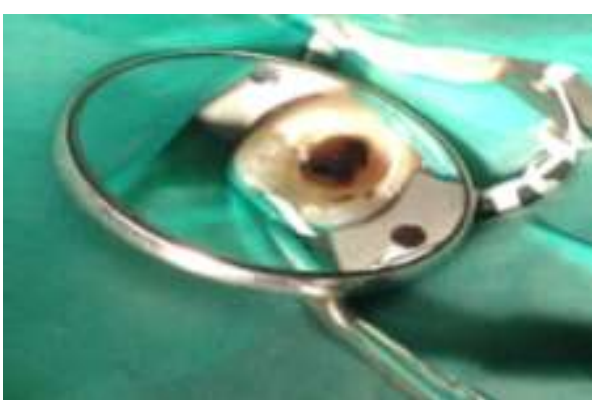

Fig 2: Preoperative IOPA 
Management of $C$ Shaped Canals in Mandibular Molars with a contemporary approach using MTA:...

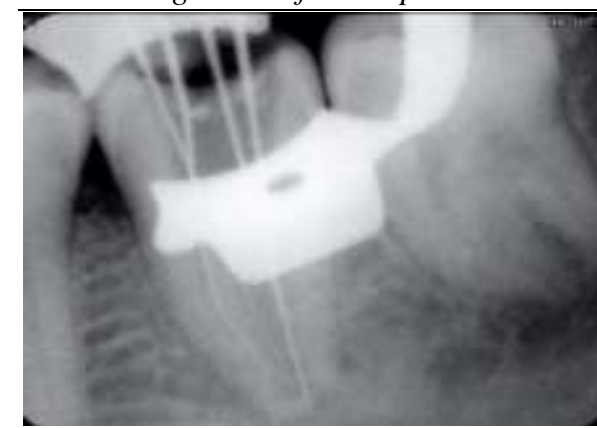

Fig 3: WL determination

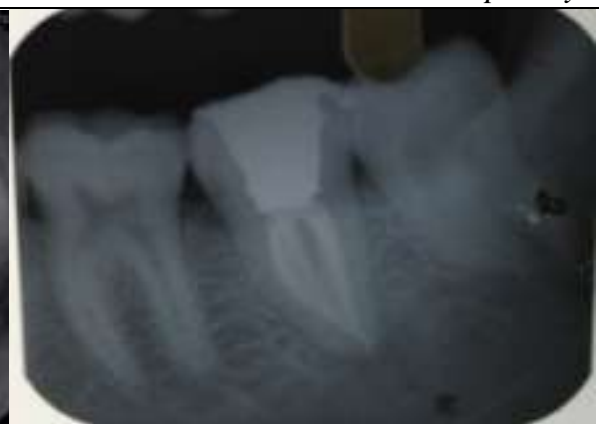

Fig 4: Post obturation IOPA

\section{Case Report II}

A 29 year old female patient reported to the department of endodontics with chief complaint of pain in relation to lower right back tooth region. Medical history of the patient was noncontributory. Patient gives history of sensitivity to thermal stimuli. Clinically there was presence of deep carious lesion in relation to 46 and 47 , also it was nonresponsive to vitality test. Radiograph revealed radiolucency extending into the pulp. Diagnosis was made as chronic periapical abcess i.r.t. 46 and chronic irreversible pulpitis with apical periodontitis i.r.t. 47. After profound anaesthesia and proper isolation, an access cavity was prepared. When the pulp chamber was explored, three orifices was recognized. Pulp tissue was extirpated and canals were cleaned and shaped using rotary hero files and irrigated using 5.25\% sodium hypochlorite. Working length was determined by using apex locator (Propex 2,Dentsply) and finally IOPA was taken. The canal was apically prepared till \#80-k file and circumferential filing (30k)was done with copious irrigation alternatively with sodium hypochlorite $2.5 \%$ and saline. Obturation was done with MTA (6 mm apical plug). For this a 80 no.guttapercha cone was checked for the fit and first bolus of MTA was placed using lumber puncture needle and was compacted with cylindrical finger pluggers and guttapercha. Moist cotton pellet was placed over the MTA. Patient was recalled after 2 days and patient was totally asymptomatic.Remaining canal was obturated with gutta-purcha with vertical compaction technique.

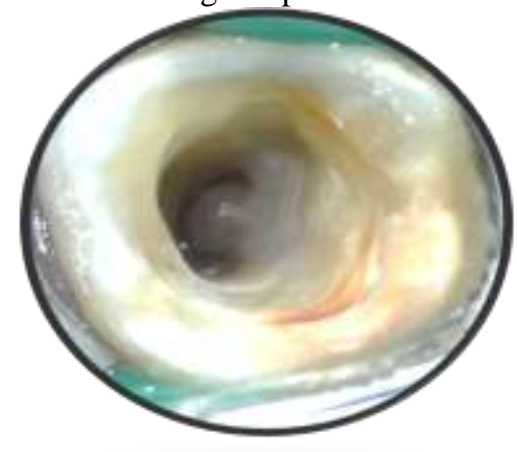

Fig 5: C shaped anatomy of pulpal chamber

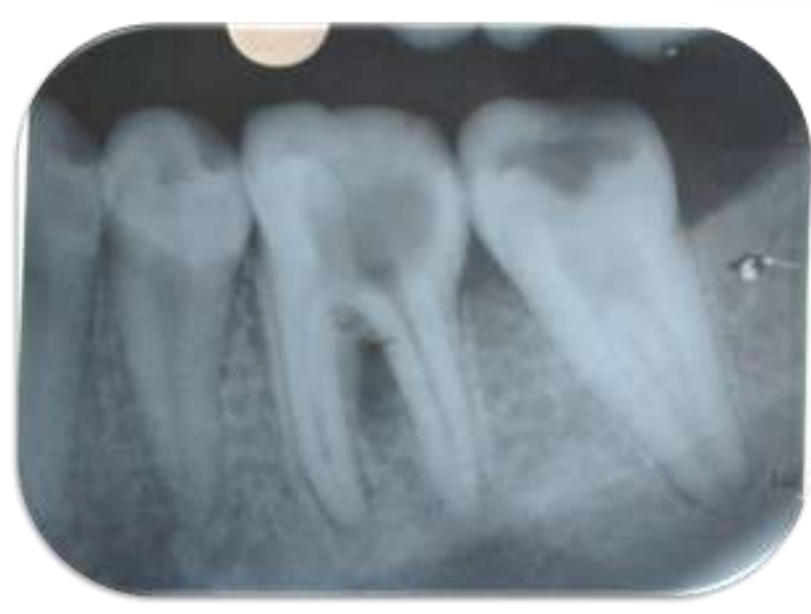

Fig 6: Preoperative IOPA

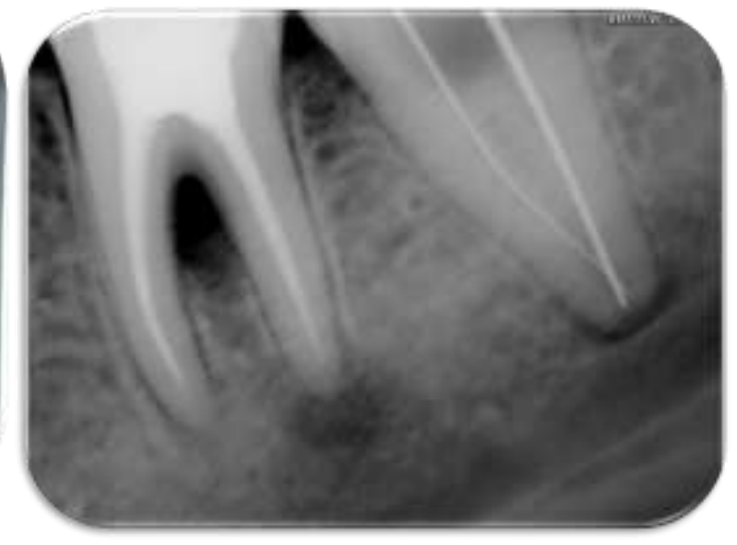

Fig 7: WL determination 


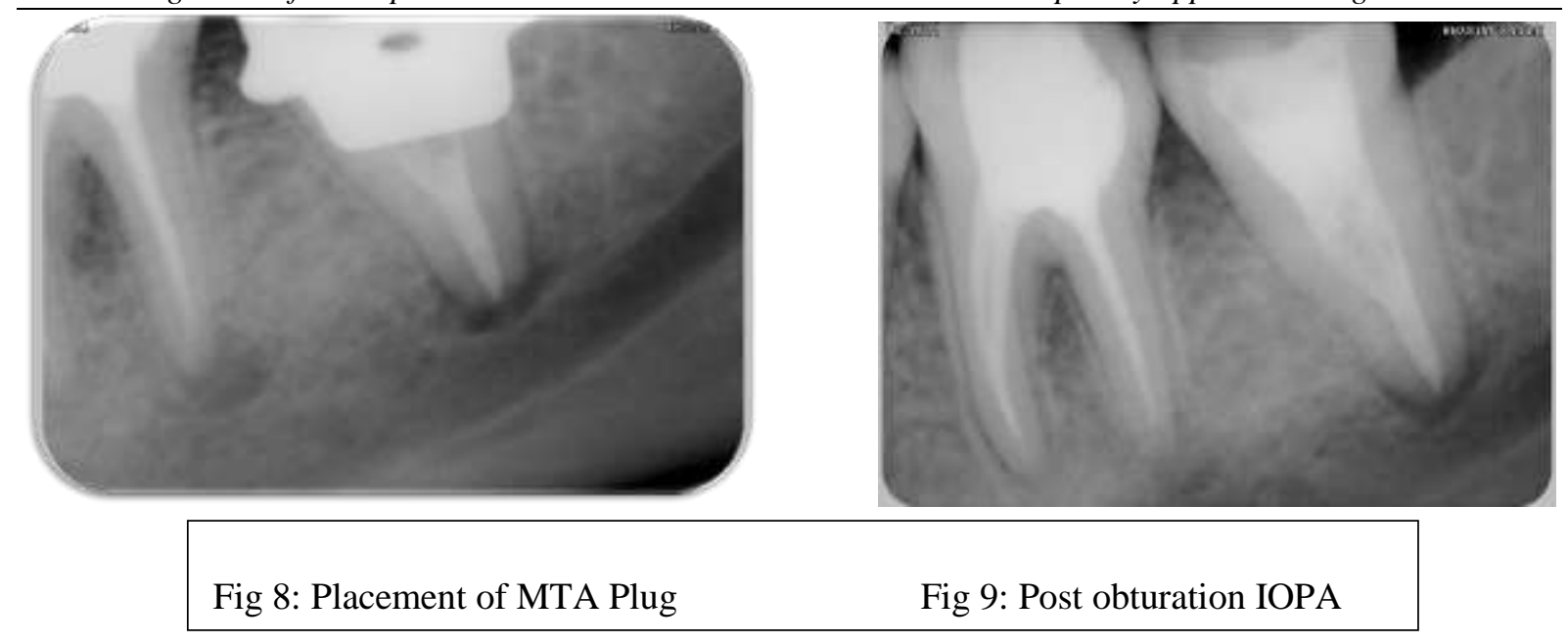

\section{DISCUSSION}

It is evident that, for endodontic treatment of teeth with C-shaped canal systems to be successful, there must be modification of procedures at all stages of the treatment, and new resources must be used. Fibre-optic transillumination can assist in the identification of the anatomy of theroot canal system. Ultrasonic instrumentation and devices for thermoplasticisation of gutta-percha assist greatly with debridement and obturation respectively. MTA is a potential apical barrier material with good sealing ability, good marginal adaptation, a high degree of biocompatibility and a reasonable setting time (about $4 \mathrm{~h}$ ).

With the MTA apical plug technique, a one-step obturation after short canal disinfection with calcium hydroxide could be performed. The application of MTA mixture should be preceded by a temporary calcium hydroxide dressing in order to limit bacterial infection in the tooth.

MTA along with MTA based sealers like MTA Fillapex are the materials which give promising results with excellent bone healing and also the sealing properties of MTA have been proven in quite a lot of studies which help in sealing the web and fin areas in the $\mathrm{C}$ shaped canals.

This technique of obturation offers effective and efficient results in apexification of teeth, allows for permanent restorations to be done in timely manner, prolonging the longevity of the teeth. Knowledge of root morphology and the configuration of the pulpal space, plays an important role in ensuring thorough debridement, and the outcome of root canal treatment.

The apical $3-5 \mathrm{~mm}$ of a root canal system is generally regarded as a critical zone, complete debridement of which has been considered as an important element for a good prognosis of root canal treatment.

The access cavity for teeth with a $\mathrm{C}$-shaped root canal system varies considerably and depends on the pulp morphology of the specific tooth. Teeth with C-shaped anatomy pose a considerable technical challenge, but the use of microscopes, sonic and ultrasonic instrumentation and plasticized obturation techniques have made treatment successful.

Knowledge of root morphology and the configuration of the pulpal space, plays an important role in ensuring thorough debridement, and the outcome of root canal treatment.

The apical $3-5 \mathrm{~mm}$ of a root canal system is generally regarded as a critical zone, complete debridement of which has been considered as an important element for a good prognosis of root canal treatment.

The access cavity for teeth with a C-shaped root canal system varies considerably and depends on the pulp morphology of the specific tooth. Teeth with C-shaped anatomy pose a considerable technical challenge, but the use of microscopes, sonic and ultrasonic instrumentation and plasticized obturation techniques have made treatment successful.

\section{CONCLUSION}

Recognition of unusual variations in the canal configurations is critical because it has been established that the root with a single tapering canal and apical foramen is the exception rather than the rule.

When sound principles of biomechanical preparation, obturation and restoration are followed, the long term prognosis for the C-shaped root retention equals that of other molars but cautious optimism would seem most appropriate when prognosticating the success of the root canal treatment of a C-shaped canal.

Due to positive studies and reports the usage of MTA in canals with periapical infections (Matt et al.2004, Bogen\&Kuttler 2009) we used this treatment protocol which gave us promising results. Such treatment protocol can prove a boon in difficult cases of $\mathrm{C}$ shaped canals.

\section{REFERENCES:-}


Management of C Shaped Canals in Mandibular Molars with a contemporary approach using MTA:...

1. Management of C Shaped Maxillary Lateral Incisor- A CaseReport- Dr.Shristhi Sharma, Dr.AnantRaghav Sharma, IOSR Journal of Dental and Medical Sciences (IOSR-JDMS)Volume 16, Issue 3 Ver. VIII (March. 2017), PP 112-115

2. The C-shaped Root Canal Configuration: A Review Hamid Jafarzadeh, DDS, MSc* JOE — Volume 33, Number 5, May 2007

3. Assessment of endodontic treatment of c-shaped root canals, Johan P Woelber, Michael Bruder, swiss dental journal VOL 124 1/2014

4. C-SHAPED CONFIGURATION OF THE ROOT CANAL SYSTEM - PROBLEMS AND SOLUTIONS Janet Kirilova, SnezhankaTopalova-Pirinska. Journal of IMAB - J of IMAB. 2014, vol. 20 , issue 1

5. Management of C Shaped Canals in Mandibular Molars: Two Case Reports 1 Rakhee Ramdas, 2 Ramesh Kumar, IOSR Journal of Dental and Medical Sciences (IOSR-JDMS) e-ISSN: 2279-0853, pISSN: 2279-0861.Volume 15, Issue 4 Ver. III (Apr. 2016),

6. C-shaped root canal system in mandibular second molars in a Chinese population evaluated by conebeam computed tomography Q. Zheng1, L. Zhan International Endodontic Journal, 44, 857-862, 2011

7. Cohen S, Burns RC. Pathways of the pulp. 8th ed. St. Louis: Mosby, 2002;196 -229.

8. Melton DC, Krell KV, Fuller MW. Anatomical and histological features of C-shaped canals in mandibular second molars. J Endod 1991;17:384-8.

9. Vertucci FJ. Root canal anatomy of the human permanent teeth. Oral Surg Oral Med Oral Pathol 1984;58:589-99.

10. Gutmann JL, Rakusin H. Perspectives on root canal obturation with thermoplasticized injectable guttapercha. IntEndod J 1987;20:261-70.

11. COOKE, H. G.; COX, F.L. C-shaped mandibular canal configurations in molars. J. Am. Dent. Assoc, v. 9. p. 836-9, Nov. 1979

12. Jou YT, Karabucak B, Levin J, Liu D. Endodontic working width: current concepts and techniques. Dental clinics of North America 2004;48:323-335.

13. Torabinejad M, Watson TF, Ford TRP (1993) Sealing ability of a mineral trioxide aggregate when used as a root end filling material. Journal of Endodontics 19, 591-5.

14. Duarte MAH, Demarchi ACCO, Yamashita JC, Kuga MC, Fraga SC (2003) PH and calcium ion release of 2 root-filling materials. Oral Surgery Oral Medicine Oral Pathology OralRadiology and Endodontics 95, 345-7.

15. Estrela C, Pesce HF (1996) Chemical analysis of the liberation of calcium and hydroxyl ions from calcium hydroxide pastes in connective tissue in the dog - part I. Brazilian Dental Journal 7, 41-6.

16. MTA preparations from different origins may vary in theirantimicrobial activityKhalid Al-Hezaimi, BDS, MSc OOOOE Volume 107, Number 5 